\title{
Solvent Effects on Structure and Reaction Mechanism: A Theoretical Study of $[2+2]$ Polar Cycloaddition between Ketene and Imine
}

\author{
Thanh N. Truong \\ Henry Eyring Center for Theoretical Chemistry, Department of Chemistry, University of Utah, \\ Salt Lake City, Utah 84112
}

Received: March 25, 1998; In Final Form: July 17, 1998

\begin{abstract}
The effects of aqueous solvent on structures and mechanism of the [2+2] cycloaddition between ketene and imine were investigated by using correlated MP2 and MP4 levels of ab initio molecular orbital theory in conjunction with the dielectric continuum Generalized Conductor-like Screening Model (GCOSMO) for solvation. We found that reactions in the gas phase and in aqueous solution have very different topology on the free energy surfaces but have similar characteristic motion along the reaction coordinate. First, it involves formation of a planar trans-conformation zwitterionic complex, then a rotation of the two moieties to form the cycloaddition product. Aqueous solvent significantly stabilizes the zwitterionic complex, consequently changing the topology of the free energy surface from a gas-phase single barrier (one-step) process to a double barrier (two-step) one with a stable intermediate. Electrostatic solvent-solute interaction was found to be the dominant factor in lowering the activation energy by $4.5 \mathrm{kcal} / \mathrm{mol}$. The present calculated results are consistent with previous experimental data.
\end{abstract}

\section{Introduction}

$[2+2]$ cycloaddition reactions are useful synthetic routes to formation of four-membered rings. From the WoodwardHoffmann rules, the $[2+2]$ reactions are thermally allowed via a supra-antara transition state. These reactions are often classified into two classes, namely, nonpolar or polar cycloadditions, having rather different mechanisms. Stereochemical and kinetic data indicate that nonpolar cycloadditions proceed via a two-step biradical mechanism with rather large activation energy. On the contrary, polar cycloadditions in solution have much lower activation energies and were suggested to have a two-step mechanism with a zwitterionic intermediate. ${ }^{1,2}$ The existence of such a stable intermediate is thought to be mainly due to solvent effects that stabilize the zwitterionic structure.

Theoretical investigations on roles of solvents in reaction mechanisms of polar cycloadditions so far have been mostly limited to applications of a simple dielectric continuum selfconsistent reaction field (SCRF) model. ${ }^{3-20}$ A general conclusion is that polar solvents stabilize the zwitterionic intermediate, consequently altering the mechanism from a concerted process in the gas phase to a stepwise one in solution via a stable zwitterionic intermediate. This is qualitatively in accord with experimental observations. However, quantitatively the magnitude of solvent effects on the free energy of activation was generally overestimated by such models. More recent detailed analysis by Lim and Jorgensen ${ }^{14}$ in comparisons to experimental data led to a conclusion that polar $[2+2]$ cycloadditions emerge as unusually challenging reactions for computational study. For instance, SCRF and the more accurate self-consistent isodensity polarizable continuum model (SCIPCM) calculations at the HF/ 6-31G* and B3LYP/6-31G* levels predict the solvent effects lowering the activation energy of the cycloaddition of 1,1dicyanoethylene (DCNE) and methyl vinyl ether (MVE) by 10$13 \mathrm{kcal} / \mathrm{mol}$ on going from $\mathrm{CCl}_{4}$ to $\mathrm{CH}_{3} \mathrm{CN}{ }^{14}$ This is substantially larger than the observed data of about $5 \mathrm{kcal} / \mathrm{mol}$.
Lim and Jorgensen have also carried out free energy perturbation (FEP) theory simulations with accurate force field that includes solute polarization effects and found even much larger solvent effects of $18-20 \mathrm{kcal} / \mathrm{mol}$ on the activation energy. It was concluded by Lim and Jorgensen ${ }^{14}$ that perhaps higher level of theory such as MCSCF with a larger basis set and more accurate continuum solvation models are required. However, it is also possible that errors were created in these FEP simulations due to double counting of the solute polarization effects-first, in the atomic charges that were calculated using dielectric continuum models, and second, implicitly in the OPLS force field. This suggestion is supported by the fact that better results were obtained by Lim and Jorgensen when gas-phase geometries and charges were used in FEP simulations.

In this study, our two main objectives are (1) to provide further analysis on the roles of solvent in structure and mechanism of polar $[2+2]$ cycloaddition and (2) to test the accuracy of the dielectric continuum generalized conductor-like screening model (GCOSMO). The GCOSMO model ${ }^{21-26}$ has been found quite successful in calculating solvation free energies, solvent effects on structures, tautomeric equilibria, vibrational spectra, and reaction profiles of proton transfer and $\mathrm{S}_{\mathrm{N}} 2$ type I and II reactions. Particularly, for chemical reactions, nearly all dielectric continuum models were able to predict correctly solvent effects on $\mathrm{S}_{\mathrm{N}} 2$ reactions, ${ }^{25,26}$ thus it would be of interest to test the dielectric continuum methodology on a more challenging reaction. For this purpose, we have investigated roles of aqueous solvent on structures and mechanism of the $[2+2]$ cycloaddition between ketene and imine. Although direct experimental data for this reaction do not exist, calculated solvent effects on structures and reactivity should be general to other $[2+2]$ polar cycloaddition reactions. The relative small size of ketene and imine allows more accurate levels of ab initio molecular orbital theory to be used, and thus provides an opportunity to focus on the accuracy of the GCOSMO solvation model. 


\section{Methodology}

The methodology for quantum modeling of the free energy profile for a reaction in solution has been discussed in detail in our previous paper. ${ }^{26}$ We provide here only a brief summary on the determination of the solution-phase free energy of activation. Let us consider a reaction ( $\mathrm{A}+\mathrm{B} \rightarrow \mathrm{TS} \rightarrow$ product) in solution. The transition state TS here is defined as the highest point on the solution-phase minimum free energy path (MFEP) that connects the reactants and product. Associated with the reactants $\mathrm{A}$ and $\mathrm{B}$ and the transition state TS are free energies of solvation denoted as $\Delta G$. The standard free energy of activation, i.e., the difference between the free energies of the transition state and reactants, in the gas phase and in solution are denoted as $\Delta G_{\mathrm{g}}^{0}$ and $\Delta G_{\mathrm{s}}^{0}$, respectively. From the thermodynamic cycle given below

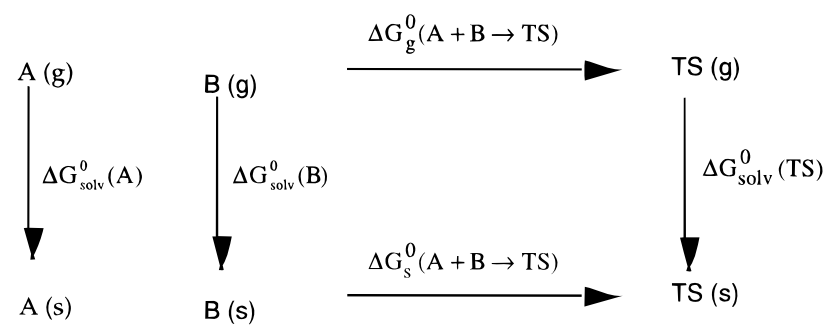

we can express the standard free energy of activation in solution as

$$
\Delta G_{\mathrm{s}}^{0}=\Delta G_{\mathrm{g}}^{0}+\left\{\Delta G_{\mathrm{solv}}^{0}(\mathrm{TS})-\left(\Delta G_{\mathrm{solv}}^{0}(\mathrm{~A})+\Delta G_{\mathrm{solv}}^{0}(\mathrm{~B})\right)\right\}
$$

where the gas-phase free energy of activation is given by

$$
\Delta G_{\mathrm{g}}^{0}=\Delta E-R T \ln \left(\frac{Q^{\mathrm{TS}}}{Q^{\mathrm{A}} Q^{\mathrm{B}}}\right)
$$

Here $\Delta E$ is the potential energy difference between the transition state and reactants; $R$ is the Boltzmann constant; $T$ is the temperature; $Q^{\mathrm{A}}, Q^{\mathrm{B}}$, and $Q^{\mathrm{TS}}$ are the gas-phase total partition functions for the reactants and transition state, respectively, evaluated with the zero of energy set at the bottom of the corresponding well on the potential energy surface. Due to the difficulties in computing the derivatives of the second term in eq 2, contribution from the solute internal degrees of freedom, it was not included in the determination of the condensed-phase transition state and reaction profile but is added as a correction. Note that eq 1 is valid only when the same geometry of the transition state is used in eq 2 . In this case, geometry of the solution-phase transition state is used.

Within the dielectric continuum methodology, we can write the free energy of solvation as

$$
\Delta G_{\mathrm{solv}}=\Delta G_{\mathrm{el}}+\Delta G_{\mathrm{dis}}+\Delta G_{\mathrm{rep}}+\Delta G_{\mathrm{cav}}
$$

where $\Delta G_{\text {el }}$ constitutes solvation terms of electrostatic nature; $\Delta G_{\text {dis }}$ and $\Delta G_{\text {rep }}$ are the solvent-solute dispersion and repulsion interactions, respectively; $\Delta G_{\text {cav }}$ is the work required to create the cavity. For polar solvents, nonelectrostatic contributions, the last three terms of eq 3 , are often small and thus were neglected in most previous dielectric continuum calculations of reactions in solution.

For the electrostatic term $\Delta G_{\text {el }}$, we used the GCOSMO model. ${ }^{21-24,27}$ This model is based on the COSMO approach originally proposed by Klamt and Schüürmann. ${ }^{28}$ It was generalized to a general description for the solute charge density without using the multipole expansion and added nonelectrostatic contributions. The essence of the COSMO and GCOSMO models is to determine first surface charges on the cavity in a conductor (the dielectric constant $\epsilon=\infty$ ) from a boundary condition that the electrostatic potential on the surface is zero. For a dielectric medium specified by the dielectric constant $\epsilon$, the corresponding surface charges are then determined approximately by scaling the screening conductor surface charge by a factor of $f(\epsilon)=(\epsilon-1) / \epsilon$ to satisfy Gauss' theorem. Note that for water $(\epsilon=78.4)$, this scaling factor is 0.987 indicating that water behaves $99 \%$ like a perfect conductor. Thus, this is a good approximation for polar solvents. When compared to the exact Poisson boundary condition, GCOSMO yields average unsigned differences in hydration free energies of less than 0.2 $\mathrm{kcal} / \mathrm{mol}$ for neutral solutes and $0.9 \mathrm{kcal} / \mathrm{mol}$ for ions. ${ }^{24}$

For the dispersion $\Delta G_{\text {dis }}$ and short-range repulsion $\Delta G_{\text {rep }}$ contributions, we adopted the Floris et al. method. ${ }^{29}$ For the cavity formation term, we employed the scaled particle fluid theory of Pierotti, ${ }^{30}$ which was transformed by Huron and Claverie $^{31}$ into an atom-molecule-type formalism. These methods have been found to be sufficiently accurate from several previous studies ${ }^{21,29,32-37}$ including our own.

\section{Computational Details}

From the Woodward-Hoffmann rule, the $[2+2]$ cycloaddition between ketene and imine is a nonconcerted process. As noted in a previous study, the concerted reaction path for polar $[2+2]$ cycloaddition does exist but has a much higher barrier even when solvent effects were included. ${ }^{5}$ For this reason, we focus only on the nonconcerted path in this study. Geometries at the stationary points, i.e., reactants, product, intermediate, and transition states for reaction both in the gas phase and in aqueous solution $(\epsilon=78.4)$ were fully optimized using the second-order Møller-Plesset perturbation theory (MP2) with the 6-31G(d,p) basis set. For the gas-phase potential surface, we further improved the accuracy of energetic information by carrying out MP4(SDTQ) single-point calculations with a slightly larger cc-pVDZ basis set at the optimized MP2 geometries. For GCOSMO calculations, the solvent excluding surface $^{37}$ was used for defining the cavity boundary. It was constructed using the GEPOL algorithm. ${ }^{39}$ The atomic radii $(\mathrm{N}: 1.74 ; \mathrm{C}: 1.64 ; \mathrm{H}: 1.17 ; \mathrm{O}: 1.58 \AA$ ) used in this study have been optimized to reproduce free energies of hydration at the room temperature for a representative set of small molecules and ions. ${ }^{24}$

All calculations were done using our locally modified Gaussian92/DFT program. ${ }^{40}$

\section{Results and Discussion}

Gas-Phase Potential Energy Surface. Optimized geometries at stationary points on both the gas-phase potential energy surface and condensed-phase free energy are shown in Figures 2-3. Relative potential energies and standard free energies are listed in Table 1. First, we found in agreement with the previous study by Assfeld et al.,,4 that the gas-phase MP2/6-31G(d,p) potential energy surface has a very shallow intermediate which is only $0.13 \mathrm{kcal} / \mathrm{mol}$ below an early transition state in the reactant channel. Both the transition state to complexation (TS1) and the complex intermediate (INT) have planar trans conformations with the $\mathrm{C}-\mathrm{N}$ distances of 1.730 and $1.598 \AA$, respectively. These structures display zwitterionic character as evidenced by their large dipole moments of 6.52 and $7.21 \mathrm{D}$, respectively (see Figures 3a,b). Both stationary points however are artifacts of the MP2/6-31G(d,p) surface. At a more accurate 


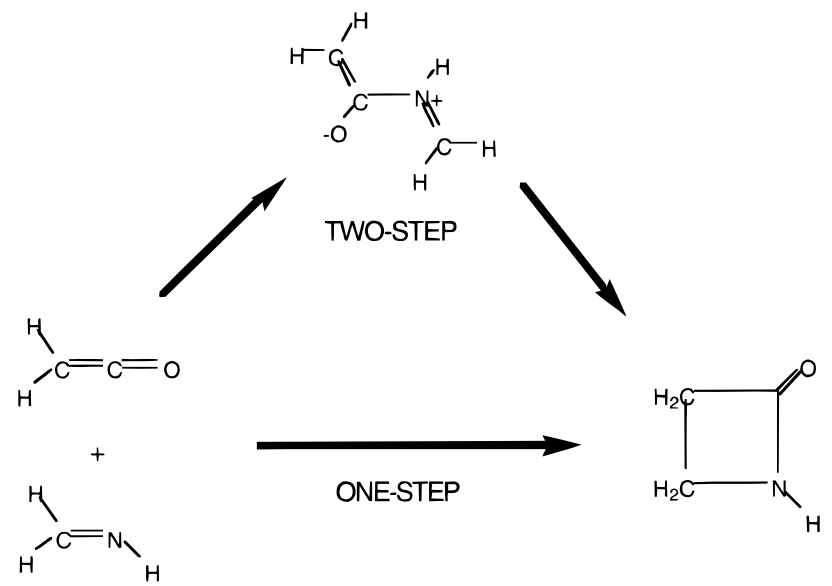

Figure 1. Possible reaction pathways for the $[2+2]$ cycloaddition of ketene and imine.
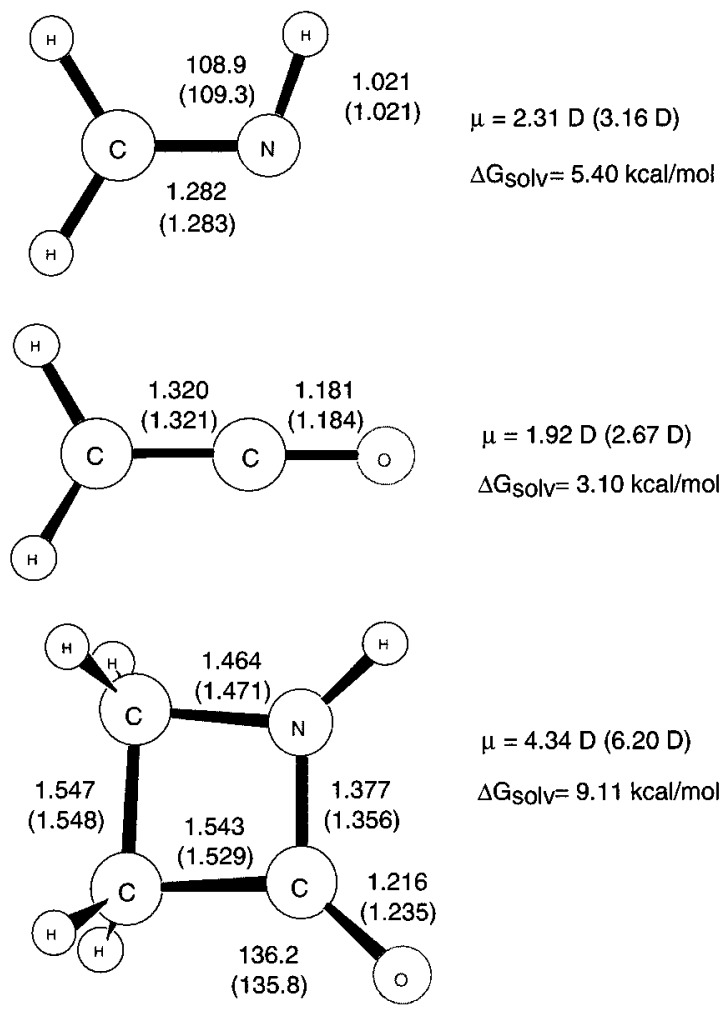

Figure 2. Geometries (distances are in $\AA$, angles are in degrees), dipole moment (Debye), and hydration free energies ( $\mathrm{kcal} / \mathrm{mol})$ for (a) imine, (b) ketene, and (c) product. Values in parentheses are for systems in aqueous solution.

level of theory, particularly the MP4(SDTQ)/cc-pVDZ level using MP2 optimized geometries, these stationary points, TS1 and INT, do not exist. Furthermore, we found that the gauche conformation for the intermediate complex does not exist at the MP2/6-31G(d,p) level, though they were reported for the cycloaddition of DNCE and hydroxyethylene at the MCSCF/ 4-31G level. Bernardi ${ }^{5}$ and Lim and Jorgensen ${ }^{14}$ also did not find such stationary points on a less accurate HF/6-31G(d) surface for the DCNE and MVE reaction. The MP4 potential surface for the cycloaddition of ketene and imine has only one transition state leading to the cycloaddition product with a barrier of $23.06 \mathrm{kcal} / \mathrm{mol}$. Adding entropic and enthalpic contributions to the potential energy differences we found that free energy surface also has a single barrier with the free energy of activation to be $37.8 \mathrm{kcal} / \mathrm{mol}$. The geometry of the transition state to cycloaddition (TS2) is shown in Figure 3c. It has a zwitterionic

(b)
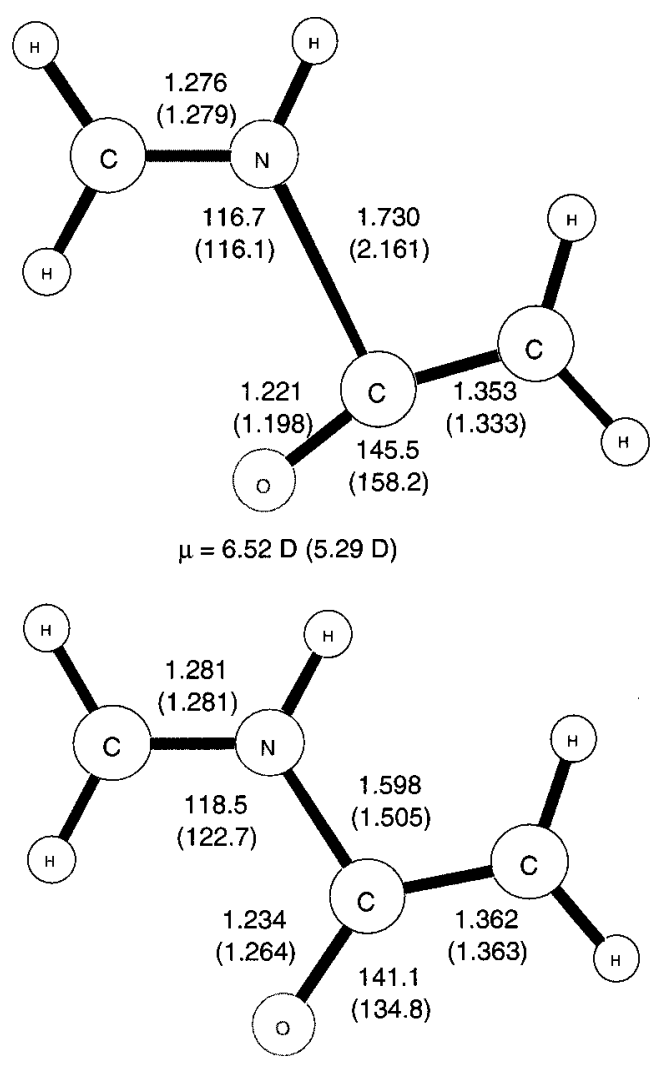

$\mu=7.21 \mathrm{D}(10.63 \mathrm{D})$

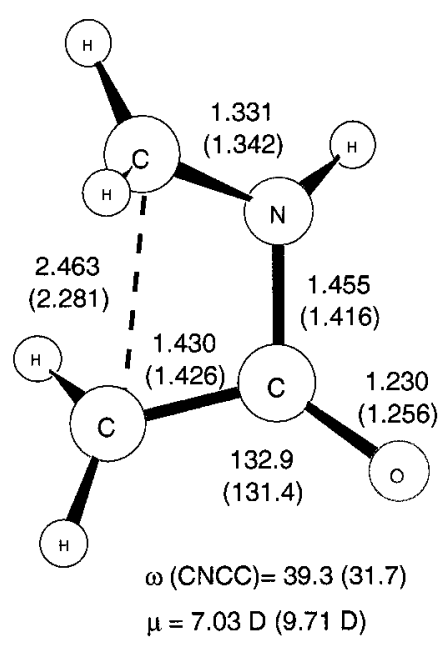

Figure 3. Similar to Figure 2, except for (a) transition state TS1, (b) intermediate INT, and (c) transition state TS2.

TABLE 1: Potential and Standard Free Energies (kcal/mol) Relative to That of Reactants

\begin{tabular}{llcccl}
\hline & \multicolumn{1}{c}{ theory/basis } & TS1 & intermediate & TS2 & product \\
\hline \multicolumn{5}{c}{ Gas Phase } \\
$\Delta E$ & MP2/6-31G(d,p) & 3.70 & 3.57 & 21.06 & -40.91 \\
& MP4/cc-pVDZ//MP2 & 5.47 & 5.93 & 23.06 & -34.52 \\
& MP2/6-31G(d) ${ }^{a}$ & 3.7 & 3.5 & 21.3 & -41.2 \\
$\Delta G^{0}$ & MP4//MP2 & 19.71 & 20.83 & 37.79 & -17.03 \\
\multicolumn{5}{c}{ Aqueous Solution } \\
$\Delta \mathrm{G}^{0}$ & GCOSMO-MP2//MP4 $^{b}$ & 16.51 & 8.24 & 33.31 & -17.38
\end{tabular}

${ }^{a}$ Reference $4 .{ }^{b}$ Our best estimates where solvation energies at the GCOSMO-MP2/6-31G(d,p) level were added to the MP4(SDTQ) gasphase single point free energies.

character with rather large Mulliken charges of -0.57 for the $\mathrm{N}$ atom of the imine and 0.66 for the connecting $\mathrm{C}$ atom of the 
ketene. The forming $\mathrm{C}-\mathrm{N}$ and $\mathrm{C}-\mathrm{C}$ bond distances are 1.56 and $2.43 \AA$, respectively, while the dihedral angle $\mathrm{CNCC}$ rotated from $180^{\circ}$ at the intermediate complex to $39^{\circ}$. These values are to compare with their corresponding parameters of the product of $1.38 \AA$ for the $\mathrm{C}-\mathrm{N}$ bond, $1.55 \AA$ for the $\mathrm{C}-\mathrm{C}$ bond, and 0 degree for the $\mathrm{CNCC}$ angle. This indicates that the reaction is quite asynchronous since at the transition state the formation of the $\mathrm{C}-\mathrm{N}$ bond is $13 \%$ to completion while it is still $57 \%$ to completion for the $\mathrm{C}-\mathrm{C}$ bond.

\section{Solvent Effects}

Structures. Aqueous solvent has little effect on the geometries of ketene and imine as shown in Figure 2. A slightly larger effect was observed for the product (see Figure 2c) where the $\mathrm{C}-\mathrm{C}$ bond distance of the ketene moiety is shortened by $0.014 \AA$, and the new $\mathrm{C}-\mathrm{N}$ bond distance is also shortened by $0.021 \AA$. However, aqueous solvent has dramatic effects on the geometries of the intermediate and both transition states TS1 and TS2 as shown in Figure $3 a-c$. This confirms the general fact that polar solvents have significant effects on structures that have a zwitterionic character. Although the transition state TS1 and intermediate INT are artifacts on the gas-phase MP2 potential surface, they are real features on the condensed-phase free energy surface, thus it is informative to discuss how solvent affects these stationary points. Aqueous solvent shifts the transition state TS1 significantly toward the entrance channel as indicated by a large increase in the $\mathrm{C}-\mathrm{N}$ bond distance from 1.73 to $2.61 \AA$ and overall shift of the ketene moiety toward its reactant geometry. Solvent effects push the intermediate INT and transition state TS2 toward the product direction as the $\mathrm{C}-\mathrm{N}$ bond distance decreases from 1.598 to $1.505 \AA$ at the intermediate and from 1.455 to $1.416 \AA$ at the transition state TS2. Similarly, solvent effects shorten the $\mathrm{C}-\mathrm{C}$ bond by $0.18 \AA$ at the transition state. The larger decrease in the $\mathrm{C}-\mathrm{C}$ bond compared to that for the $\mathrm{C}-\mathrm{N}$ bond indicating that solvent effects reduce asynchrony in this cycloaddition reaction. Changes in angles and dihedral angle toward the product values are also noticeable and are consistent with the above observation. It is interesting to point out that such effects of water solvent on the structures of the intermediate and transition state are unexpected since it has been known that polar solvents promote the charge separation by increasing the zwitterionic bond distance to gain favorable solvation energy. Despite the fact that the zwitterionic $\mathrm{C}-\mathrm{N}$ bond at the transition state TS2 is shortened upon transfer from vacuum to water, the dipole moment is increased noticeably by $2.5 \mathrm{D}$. In fact, the calculated dipole moment for the transition state TS2 of $9.71 \mathrm{D}$ agrees well with experimental estimates $^{41}$ of $10-14$ D for such transition states. Similar although smaller solvent effects were observed in a previous study by Assfeld et al. ${ }^{4}$ This may be due to (1) the use of an ellipsoidal cavity in previous SCRF calculations and (2) the fact that calculations were done for less polar solvents $(\epsilon=2.21$ and 37.5).

Energetics and Mechanism. Schematic free energy profiles for the ketene and imine cycloaddition reaction in both the gas phase and in aqueous solution are shown in Figure 4. At room temperature, the gas-phase free energy profile exhibits a single barrier with the calculated free energy of activation of $37.8 \mathrm{kcal} /$ $\mathrm{mol}$ and the reaction free energy of $-17.03 \mathrm{kcal} / \mathrm{mol}$. Solvent was found to have little effect on the later property. It lowers the reaction free energy by only $0.35 \mathrm{kcal} / \mathrm{mol}$. Aqueous solvent, however, has a much larger effect in the entrance channel. It stabilizes the zwitterionic complex. As a consequence, in aqueous solution the free energy profile changes from

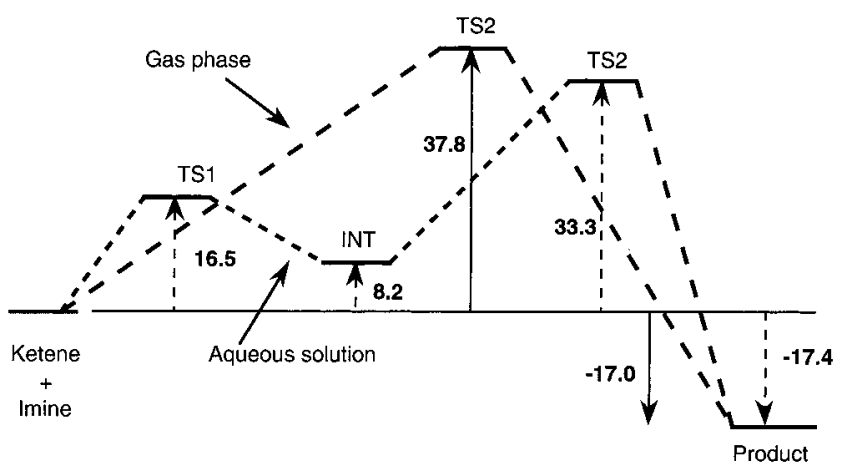

Figure 4. Schematic free energy profiles for the $[2+2]$ cycloaddition of ketene and imine in the gas phase and in aqueous solution. Standard free energies, $\Delta G$, at the stationary points are given for the gas phase (near the solid arrows) and aqueous solution (near the dashed arrows).

a single barrier to a double barrier separated by a stable intermediate. The free energy of activation for complexation is $16.51 \mathrm{kcal} / \mathrm{mol}$ while it is $33.31 \mathrm{kcal} / \mathrm{mol}$ for cycloaddition. Thus, aqueous solvent lowers the free energy of activation by $4.48 \mathrm{kcal} / \mathrm{mol}$ and consequently enhances the rate of cycloaddition by about a factor of 1835 at the room temperature. This is in a reasonable range for polar $[2+2]$ cycloadditions. ${ }^{42}$ Nonelectrostatic contributions were found to increase the activation energy by $0.1 \mathrm{kcal} / \mathrm{mol}$. Thus, electrostatic solventsolute interaction is a dominant factor in such a lowering of the activation energy. The stable intermediate complex is 8.24 $\mathrm{kcal} / \mathrm{mol}$ higher in free energy compared to the reactants. Our results confirm the two-step mechanism suggested much earlier by Huisgen and co-workers and are consistent with experimental data where such complexes were found stable for some time at room temperature in a polar matrix. ${ }^{43}$ It is important to point out that the characteristic motions along the reaction path for both the gas-phase and condensed-phase are similar. From the reactant channel, first the reactants approach each other in a planar trans conformation in a process to preserve the $\pi$-conjugation of the complex and thus promoting charge transfer from the imine to the ketene moiety. Then the two moieties rotate to form the cycloaddition product. Aqueous solvent stabilizes the zwitterionic complex at the end of the first stage, thus creating a stable well and a small barrier in the entrance channel. In other words, aqueous solvent changes the topology of the free energy surface and thus the kinetics of this reaction but does not change the characteristic motions along the reaction path. However, for substituted ketene and imine reaction, the potential energy surface is much more complex. Here the magnitude of solvent effects is different for different directions of attack of imine (syn or anti) to the ketene, thus leads to different diastereoisomers. ${ }^{20}$

\section{Conclusion}

Progress in theoretical chemistry has allowed accurate incorporation of solvent effects in studying structure and mechanisms of reactions in solution. In this study, we have investigated aqueous solvent effects on the $[2+2]$ cycloaddition of ketene and imine using accurate ab initio molecular orbital theory with the GCOSMO dielectric continuum solvation approach. We found that at room temperature, gas-phase reaction proceeds via a one-step (single-barrier) mechanism. Aqueous solvent substantially stabilizes the intermediate zwitterionic complex leading to a two-step mechanism. Despite large effects of the aqueous solvent on the topology of the free energy surface, the characteristic motions along the reaction path 
are similar. In particular, reaction proceeds first via a planar trans conformation to preserve $\pi$-conjugation, then rotates to yield the cycloaddition product. Solvent effects were found to lower the free energy of activation by $4.48 \mathrm{kcal} / \mathrm{mol}$, and that is consistent with previous experimental data. Electrostatic solvent-solute interaction was found to be the dominant factor in the overall solvent effects. Combined with our earlier success in studying solvent effects on structures and mechanisms of other reactions, this study has added further confidence in the ability of the GCOSMO dielectric continuum model to model reactions in solution.

Acknowledgment. This work was supported in part by the National Science Foundation via a National Young Investigator Award.

\section{References and Notes}

(1) Huisgen, R. Acc. Chem. Res. 1977, 10, 117.

(2) Huisgen, R. Pure Appl. Chem. 1980, 52, 2283.

(3) Assfeld, X.; Sordo, J. A.; Gonzalez, J.; Ruizlopez, M. F.; Sordo, T. L. THEOCHEM 1993, 106, 193.

(4) Assfeld, X.; Ruiz-López, M. F.; Gonzalez, J.; López, R.; Sordo, J. A.; Sordo, T. L. J. Comput. Chem. 1994, 15, 479.

(5) Bernardi, F.; Pappalardo, R. R.; Robb, M. A.; Venturini, A. THEOCHEM 1995, 357, 33-36.

(6) Cossio, F. P.; Roa, G.; Lecea, B.; Ugalde, J. M. J. Am. Chem. Soc. 1995, 117, 12306

(7) Domingo, L. R.; Picher, M. T.; Andres, J.; Moliner, V.; Safont, V. S. Tetrahedron 1996, 52, 10693-10704.

(8) Essefar, M.; El Mouhtadi, M.; Liotard, D.; Abboud, J.-L. M. J. Chem. Soc., Perkin Trans. 2 1988, 143.

(9) Fang, D.; Fu, X. Chem. Phys. Lett. 1996, 259, 265-270.

(10) Karcher, T.; Sicking, W.; Sauer, J.; Sustmann, R. Tetrahedron Lett. 1992, 33, 8027-8030.

(11) Lecea, B.; Arrieta, A.; Roa, G.; Ugalde, J. M.; Cossio, F. P. J. Am. Chem. Soc. 1994, 116, 9613-9619.

(12) Lecea, B.; Arrieta, A.; Lopez, X.; Ugalde, J. M.; Cossio, F. P. J. Am. Chem. Soc. 1995, 117, 12314-12321.

(13) Lecea, B.; Arrastia, I.; Arrieta, A.; Roa, G.; Lopez, X.; Arriortua,

M. I.; Ugalde, J. M.; Cossio, F. P. J. Org. Chem. 1996, 61, 3070-3079.

(14) Lim, D.; Jorgensen, W. L. J. Phys. Chem. 1996, 100, 17490-17500.

(15) Lopez, R.; Suarez, D.; Ruiz-Lopez, M. F.; Gonzalez, J.; Sordo, J.

A.; Sordo, T. L. J. Chem. Soc., Chem. Commun. 1995, 16, 1677-1678.

(16) Rastelli, A.; Bagatti, M.; Gandolfi, R. J. Am. Chem. Soc. 1995, $117,4965-4975$.
(17) Reguero, M.; Pappalardo, R. R.; Robb, M. A.; Rezpa, H. S. J. Chem. Soc., Perkin Trans. 2 1993, 8, 1499-1503.

(18) Sperling, D.; Mehlhorm, A.; Reissig, H. U.; Fabian, J. Liebigs Ann. Chem. 1996, 1615-1621.

(19) Sustmann, R.; Sicking, W. Tetrahedron 1992, 48(47), 1029311300

(20) Lopez, R.; Ruiz-Lopez, M. F.; Rinaldi, D.; Sordo, J. A.; Sordo, T. L. J. Phys. Chem. 1996, 100, 10600.

(21) Truong, T. N.; Stefanovich, E. V. Chem. Phys. Lett. 1995, 240, 253.

(22) Truong, T. N.; Stefanovich, E. V. J. Chem. Phys. 1995, 103, 3709.

(23) Truong, T. N.; Nguyen, U. N.; Stefanovich, E. V. Int. J. Quantum Chem. 1996, 60, 1615. 65

(24) Stefanovich, E. V.; Truong, T. N. Chem. Phys. Lett. 1995, 244,

(25) Truong, T. N.; Stefanovich, E. V. J. Phys. Chem. 1995, 99, 14700.

(26) Truong, T. N.; Truong, T.-T. T.; Stefanovich, E. V. J. Chem. Phys. 1997, 107,1881 .

(27) Stefanovich, E. V.; Truong, T. N. J. Chem. Phys. 1996, 105, 2961.

(28) Klamt, A.; Schüürmann, G. J. Chem. Soc., Perkin Trans. 2 1993, 799.

(29) Floris, F. M.; Tomasi, J.; Ahuir, J. L. P. J. Comput. Chem. 1991 12,784

(30) Pierotti, R. A. Chem. Rev. 1976, 76, 717.

(31) Huron, M. J.; Claverie, P. J. Phys. Chem. 1972, 76, 2123.

(32) Aguilar, M. A.; Olivares del Valle, F. J. Chem. Phys. 1989, 138, 327.

(33) Floris, F.; Tomasi, J. J. Comput. Chem. 1989, 10, 616.

(34) Floris, F. M.; Tani, A.; Tomasi, J. Chem. Phys. 1993, 169, 11.

(35) Frecer, V.; Miertus, S.; Majekova, M. THEOCHEM 1991, 73, 157.

(37) Rinaldi, D.; Cabral, B. J. C.; Rivail, J. L. Chem. Phys. Lett. 1986 , 125,495 .

(38) Richards, F. M. Annu. Rev. Biophys. Bioeng. 1977, 6, 151.

(39) Pascual-Ahuir, J. L.; Silla, E.; Tuñon, I. J. Comput. Chem. 1994, 15,1127

(40) Frisch, M. J.; Trucks, G. W.; Schlegel, H. B.; Gill, P. M. W.; Johnson, B. G.; Wong, M. W.; Foresman, J. B.; Robb, M. A.; Head-Gordon, M.; Replogle, E. S.; Gomperts, R.; Andres, J. L.; Raghavachari, K.; Binkley, J. S.; Gonzalez, C.; Martin, R. L.; Fox, D. J.; Defrees, D. J.; Baker, J.; Stewart, J. J. P.; Pople, J. A. G92/DFT; G.3 ed.; Gaussian, Inc.: Pittsburgh, 1993.

(41) Steiner, G.; Huisgen, R. J. Am. Chem. Soc. 1973, 95, 5056.

(42) Reichardt, C. Solvents and Solvent Effects in Organic Chemistry; VCH Publishers: New York, 1990.

(43) Pacansky, J.; Chang, J. S.; Brown, D. W.; Schwarz, W. J. J. Org. Chem. 1982, 47, 2233. 